\title{
Valores de latencia y respuestas electrofisiológicas de los diferentes componentes de los potenciales evocados auditivos del tallo cerebral en mono rhesus a diferentes intensidades (70-30 $\mathrm{dB})$
}

\author{
Latency values and electrophysiological responses of the different components \\ of the brainstem auditory evoked potentials in rhesus monkeys at \\ different intensities (70-30 dB)
}

\author{
A Ibáñez-Contreras ${ }^{a, b *}$, B Hernández-Godínez ${ }^{a, b}$, A Durand-Rivera ${ }^{c}$ \\ ${ }^{a}$ Centro de Investigación, Proyecto CAMINA, A.C., Ciudad de México, México D.F. \\ bBIOINVERT®, Biología Integral para Vertebrados, Unidad de Experimentación Animal, Estado de México, México. \\ 'Laboratorio de Neuroprotección, Instituto Nacional de Rehabilitación, Ciudad de México, México D.F.
}

\begin{abstract}
SUMMARY
The records of the Brainstem Auditory Evoked Potentials (BAEPs) are sensitive to differences in stimulation parameters, conditions of registration and characteristics of the species, so it is essential to have normal values for each laboratory. The aim of our study was to obtain normative data on the latency values and electrophysiological responses of the different components of the BAEPs in the rhesus monkey, at different intensities (70-30 dB). This study was performed using 14 non-human primates of the Macaca mulatta species, with an average age of 7 years, allotted into two groups of 7 males with an average weight of $7 \mathrm{~kg}$ and 7 females weighing approximately $5 \mathrm{~kg}$. The BAEP were obtained by the stimulation of the ear with rarefaction "clicks" of 70, 50 and $30 \mathrm{~dB}$ of intensity. The cerebral electric activity was picked up by gold disc electrodes, placed in the Cz (+), A1, A2 (-) and Fz derivations as land, according to the 10/20 international system. Four waves were observed in males and females at the different intensities used. Since no significant differences were found in the Student t-test by separated afferences, afferents were unified, evaluating the absolute latencies of the four waves obtained and the intervals I-II, II-IV and I-IV in the 14 subjects, at different intensities.
\end{abstract}

Palabras clave: latencias, potenciales evocados auditivos de tallo cerebral (PEATC), latencias interondas.

Key words: latencies, brainstem's auditory evoked potentials (BAEP), interpeak latencies.

\section{INTRODUCCIÓN}

Los Potenciales Evocados Auditivos de Tronco Cerebral (PEATC) representan las respuestas bioeléctricas generadas a partir de un estímulo acústico transitorio en el sistema auditivo nervioso central a la altura del tronco cerebral (Soares y col 2010, DiPietro y col 2010). Una de las aplicaciones clínicas más difundidas de los PEATC ha sido su empleo como indicador funcional para la evaluación objetiva de la audición en diversas especies.

En los últimos años se han publicado diversos trabajos que buscan conocer y estandarizar los valores normales de las latencias de los PEATC en diferentes especies de primates no humanos (PNH) (Allen y Starr 1978, Doyle y col 1983, Velasco y col 1984, Møller y Burgess 1986, Pineda y col 1989, Lasky y col 1995, Laughlin y col 1999, Torre y Fowler 2000, Torre y col 2000, Alegre y col 2001, Torre y col 2004, Fowler y col 2010, Ibáñez-Contreras y col

Aceptado: 19.07.2012.

* Calz. de Tlalpan 4430 Col. Toriello Guerra, 14050, Mexico D.F. México; aibanez.2104@gmail.com
2011). Sin embargo, aunque existen estos estudios, para la obtención de un registro correcto y confiable es necesario conocer los valores de normalidad de las latencias de los principales componentes y su relación con las distintas intensidades en cada laboratorio (Delgado y col 2003, Arai 2008, Soares y col 2010, Ibáñez-Contreras y col 2011), debido a la gran variabilidad de los resultados obtenidos a partir de los PEATC en relación a los parámetros de registro (Elías 1996, McFadden y col 1999, Yadav y col 2002, Delgado y col 2003, Henry 2004, Hultcrantz y col 2006, Ibáñez-Contreras y col 2011). Es por esto que el objetivo de este trabajo es obtener datos normativos acerca de los valores de latencia y de las respuestas electrofisiológicas de los diversos componentes auditivos a través de los intervalos interonda, a partir de los PEATC en mono rhesus, utilizando diferentes intensidades (70-30 dB).

\section{MATERIAL Y MÉTODOS}

Los sujetos de estudio fueron PNH de la especie Macaca mulatta, alojados en condiciones de cautiverio en el Centro de Investigación Proyecto CAMINA para Curar la Parálisis A.C. en México D.F. C.P. 1405. La unidad 
de PNH del Centro de Investigación Proyecto CAMINA A.C. está dada de alta y registrada con el número DGVSPIMVS-CR-IN-1014-D.F./08 ante la Secretaría de Medio Ambiente y Recursos Naturales (SEMARNAT 2007). Se utilizaron 14 monos rhesus jóvenes (M. mulatta), con una edad aproximada de siete años, siete machos con un peso aproximado de $8 \mathrm{~kg}$, y siete hembras con un peso aproximado de $5 \mathrm{~kg}$.

La investigación se realizó de acuerdo a la NOM-062ZOO-1999 (Norma Oficial Mexicana 1999), así como la normatividad internacional del Código Zoosanitario Internacional de la Organización Mundial de la Salud (OMS) y fue aprobada por el Comité Interno de Cuidado y Uso de Animales de Laboratorio (CICUAL) y por las comisiones de Ética e Investigación del Centro de Investigación Proyecto CAMINA, A.C. Los animales fueron sedados con Tiletamina-Zolacepam (Zoletil®; Virbac Laboratories, Carros, France) en una dosis de $4 \mathrm{mg} / \mathrm{kg}$, por vía intramuscular (Galván-Montaño y col 2010, Ibáñez-Contreras y col 2011).

Los PEATC se realizaron en el Laboratorio de Neuroprotección del Instituto Nacional de Rehabilitación (INR), utilizando una computadora para potenciales marca Viasis Healthcare Niccolet ${ }^{\circ}$ (Viasys Healthcare, Conshokocken, PA, USA), a la cual se le programaron los filtros pasabanda que permitieron el paso de frecuencias entre 100 y $3.000 \mathrm{~Hz}$, con una sensibilidad de $10 \mu \mathrm{V}$, promediando 2.000 estímulos. La estimulación de los oídos fue con "clicks" o chasquidos de rarefacción (polaridad negativa) a 70, 50 y $30 \mathrm{~dB}$ de intensidad, con una duración de $0,1 \mathrm{msec}$; dichos "clicks" fueron liberados a través de los audífonos que forman parte del equipo. El oído contralateral fue enmascarado con ruido blanco a $20 \mathrm{~dB}$ debajo de la intensidad explorada. La impedancia de los electrodos se mantuvo por debajo de los $5 \mathrm{k} \Omega$. Para la obtención del registro de actividad eléctrica se realizó una serie descendente de estimulación a tres diferentes intensidades $(70 \mathrm{~dB}, 50 \mathrm{~dB}, 30 \mathrm{~dB})$ comenzando la estimulación en $70 \mathrm{~dB}$ y terminando en $30 \mathrm{~dB}$. Se obtuvieron 2.000 promediaciones para cada nivel de estimulación y la respuesta fue replicada por lo menos una vez para asegurar la reproducibilidad de los eventos (cuadro 1). Se estimuló primero el oído izquierdo y a continuación el derecho. Los animales fueron rasurados y se limpió el área con agua y jabón neutro para reducir la impedancia. La actividad eléctrica cerebral se recogió por medio de electrodos de disco colocados en A1 y A2 (-) y el vértice del cráneo $\mathrm{Cz}(+)$, y finalmente $\mathrm{Fp}$ (frontopolar) como tierra, según el sistema 10/20 internacional (American Encephalographic Society 1994, Ibáñez-Contreras y col 2011).

\section{ANÁLISIS ESTADÍSTICO}

El análisis estadístico se realizó por medio del programa SPSS versión 17,0 para Windows (SPSS, Chicago, IL).
Cuadro 1. Parámetros de estimulación y registro. Stimulation and recording parameters.

\begin{tabular}{ll}
\hline \multicolumn{2}{c}{ Parámetros de estimulación } \\
\hline Tipo & Click \\
Duración & $0,1 \mathrm{msec}$ \\
Intensidad & $70-30 \mathrm{~dB} \mathrm{nHL}$ \\
\hline \multicolumn{2}{c}{ Parámetros de registro } \\
\hline Colocación de electrodos & $\mathrm{Cz}-\mathrm{Fz}-\mathrm{A} 1-\mathrm{A} 2$ \\
Filtro de paso alto & $3000 \mathrm{~Hz}$ \\
Filtro de paso bajo & $100 \mathrm{~Hz}$ \\
Sensibilidad & $10 \mu \mathrm{V}$ \\
Impedancia & $<5 \mathrm{k} \Omega$ \\
$\mathrm{N}^{\mathrm{o}}$ de promediaciones & 2.000 \\
Registros por intensidad & 2 \\
\hline
\end{tabular}

Se obtuvieron medidas de tendencia central, medias, y desviación estándar, en las diferentes ondas a 70, 50 y $30 \mathrm{~dB}$. Se realizó estadística paramétrica (comparación de medias) utilizando la prueba " $t$ " de Student para comparar la latencia de las ondas I, II, III y IV, para machos y para las hembras. Posteriormente con la misma prueba se compararon ambas aferencias (derecha-izquierda) sin reportarse diferencias estadísticamente significativas, por lo que se unificaron las aferencias.

\section{RESULTADOS}

Se observaron cuatro ondas constantes y definidas en los primeros $10 \mathrm{mseg}$, tanto en machos como en hembras, en las diferentes intensidades empleadas, 70,50 y $30 \mathrm{~dB}$. En la figura 1 se muestra la morfología de las ondas tanto en machos como en hembras, observándose cambios en la amplitud de las ondas en relación a la intensidad utilizada; en ambos casos el componente más estable e identificable hasta $30 \mathrm{~dB}$ fue la onda IV. En el cuadro 2 se muestra el promedio obtenido de las latencias en aferencias unidas para cada onda (I, II, III, IV) en las diferentes intensidades (70-30 dB) evaluadas tanto en machos como en hembras; se pueden apreciar valores más alargados en el caso de los machos en relación a las hembras. Se observa en ambos casos, tanto en machos como en las hembras, que a $70 \mathrm{~dB}$ las latencias obtenidas son más pequeñas en comparación a los valores obtenidos a $30 \mathrm{~dB}$. Las latencias interonda de las ondas I, II y IV se muestran en la cuadro 3 para cada intensidad, observándose que independientemente de la intensidad utilizada los valores son estables. En el caso de los machos de igual forma se observa un alargamiento principalmente en los intervalos II-IV y I-IV, obteniéndose valores de 2,03 mseg (II-IV) y de 2,93 mseg (I-IV) en relación a los valores obtenidos en las hembras, $1,54 \mathrm{mseg}$ (II-IV) y 2,55 mseg (I-IV). 


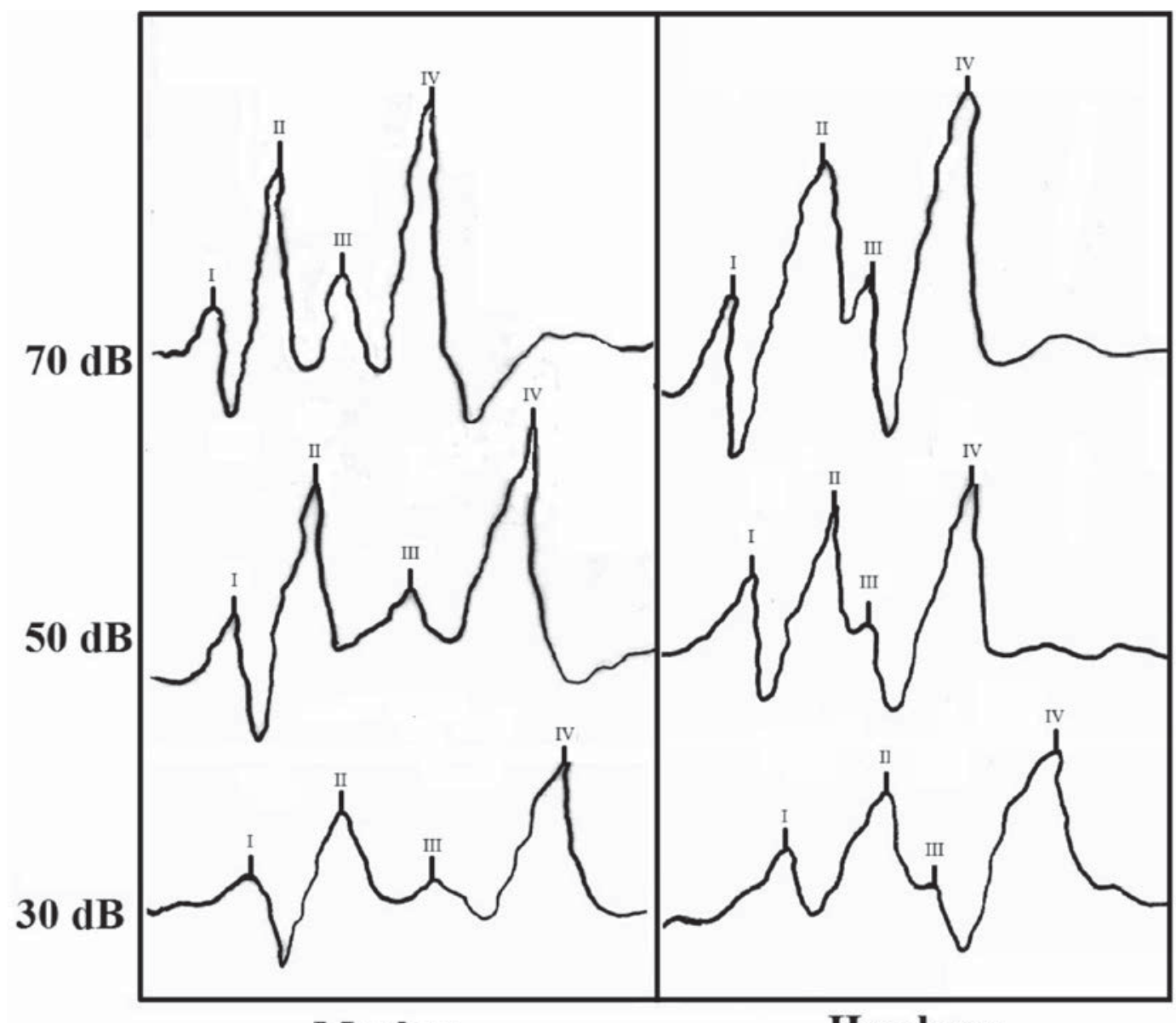

\section{Machos}

Hembras

Figura 1. Morfología de las cuatro ondas obtenidas a partir de los PEATC (I, II, III y IV), en machos y hembras a diferentes intensidades $(70,50$ y $30 \mathrm{db})$.

Morphology of the 4 waves obtained from the BAEPs (I, II, III and IV), in males and females at different intensities (70, 50 and $30 \mathrm{db})$.

Cuadro 2. Latencias absolutas (media \pm DE) de las ondas I, II, III y IV de machos $(n=7)$ y hembras $(n=7)$, aferencias unidas, en las diferentes intensidades.

Values of absolute latencies (mean \pm SD) of waves I, II, III and IV of males $(n=7)$ and females $(n=7)$, united afferents, at different intensities.

\begin{tabular}{cccc}
\hline Intensidad $(\mathrm{dB})$ & Machos $(\mathrm{n}=7)$ & & Hembras $(\mathrm{n}=7)$ \\
\cline { 2 - 2 } & Media $\pm \mathrm{DE}$ & & Media \pm DE \\
\hline $\mathrm{I}_{70}$ & $1,91 \pm 0,11$ & & $1,63 \pm 0,08$ \\
$\mathrm{I}_{50}$ & $2,16 \pm 0,23$ & & $2,05 \pm 0,12$ \\
$\mathrm{I}_{30}$ & $2,79 \pm 0,15$ & & $2,78 \pm 0,13$ \\
II $_{70}$ & $2,79 \pm 0,09$ & & $2,60 \pm 0,10$ \\
II $_{50}$ & $3,06 \pm 0,22$ & & $3,04 \pm 0,11$ \\
II $_{30}$ & $3,70 \pm 0,15$ & & $3,81 \pm 0,13$ \\
III $_{70}$ & $3,63 \pm 0,25$ & & $3,41 \pm 0,20$ \\
III $_{50}$ & $4,06 \pm 0,23$ & & $3,78 \pm 0,15$ \\
III $_{30}$ & $4,69 \pm 0,35$ & & $4,44 \pm 0,14$ \\
$\mathrm{IV}_{70}$ & $4,84 \pm 0,10$ & & $4,16 \pm 0,09$ \\
IV $_{50}$ & $5,10 \pm 0,23$ & & $4,61 \pm 0,12$ \\
$\mathrm{IV}_{30}$ & $5,73 \pm 0,15$ & & $5,36 \pm 0,12$ \\
\hline
\end{tabular}

Cuadro 3. Promedio de las latencias interonda I-II, II-IV y I-IV para machos $(n=7)$ y hembras $(n=7)$ en las distintas intensidades.

Interpeak latencies average I-II, II-IV y I-IV for males $(n=7)$ and females $(n=7)$ at different intensities.

\begin{tabular}{cccc}
\hline $\begin{array}{c}\text { Intervalo interonda } \\
\text { Machos }\end{array}$ & $70 \mathrm{~dB}$ & $50 \mathrm{~dB}$ & $30 \mathrm{~dB}$ \\
\hline I-II & 0,93 & 0,95 & 0,93 \\
II-IV & 2,04 & 2,03 & 2,03 \\
I-IV & 2,93 & 2,93 & 2,92 \\
\hline Intervalo interonda & $70 \mathrm{~dB}$ & $50 \mathrm{~dB}$ & $30 \mathrm{~dB}$ \\
Hembras & & & \\
\hline I-II & 0,92 & 0,91 & 0,92 \\
II-IV & 1,55 & 1,54 & 1,54 \\
I-IV & 2,53 & 2,56 & 2,55 \\
\hline
\end{tabular}




\section{DISCUSIÓN}

Los PNH son un modelo animal ideal para el estudio de los mecanismos neurales fundamentales en la función auditiva (Populin 2006), debido a su cercanía filogenética con el humano. Por esta razón se han realizado diversos trabajos evaluando la funcionalidad de la vía auditiva a través de los PEATC en diversas especies de PNH (Velasco y col 1984, Møller y Burgess 1986, Pineda y col 1989, Kamada y col 1991, Doyle y Webster 1991, Lasky y col 1995, Laughlin y col 1999, Alegre y col 2001, Torre y col 2004, Rhode y col 2010, Fowler y col 2010, IbáñezContreras y col 2011); sin embargo, las condiciones y los parámetros de registro en estos estudios no fueron los mismos, lo que dificulta el empleo de estos valores de referencia para otros laboratorios.

En este trabajo se observaron cuatro ondas constantes y definidas en las diferentes intensidades utilizadas (70, 50 y $30 \mathrm{~dB}$ ), tanto en machos como en hembras (figura 1), tal como lo indican Møller y Burgess (1986), Lasky y col (1995), Laughlin y col (1999), Recanzone (2000), Alegre y col (2001) e Ibáñez-Contreras y col (2011), en donde describen la aparición de cuatro ondas en los primeros 10 mseg en monos rhesus (Macaca mulatta). Las medias obtenidas de las latencias absolutas de las cuatro ondas registradas en el caso de los machos se observan más alargadas en relación a las latencias de las hembras (cuadro 2). Esto ha sido atribuido al dimorfismo sexual, siendo el tamaño del cráneo, la temperatura corporal y los efectos hormonales los causantes de estas variaciones (Bast y col 1961, Schneiderman 1992, Ankel-Simons 2000, Ibáñez-Contreras y col 2011).

En humanos está ampliamente descrito que las ondas más representativas y de las cuales se obtienen los intervalos interonda de los PEATC son la I, III y V (Elías 1997, López-Escámez y col 1999, Gil-Carcedo y col 2004, Soares y col 2010, DiPietro y col 2010). En contraste, los estudios realizados en diferentes especies de $\mathrm{PNH}$ reportan diversos resultados en cuanto a la asignación de los generadores neuronales de la vía auditiva, describiendo diferentes cantidades de ondas, lo que dificulta la estandarización de los PEATC. Allen y Starr (1976) describen la aparición de cinco ondas en monos rhesus anestesiados; sin embargo, consideran que sólo las primeras cuatro ondas son equivalentes a las cinco ondas reportadas en humanos, esto con base al valor de las latencias, así como por su ubicación anatómica. Por otro lado, Velasco y col (1984) reportan la presencia de siete ondas en monos rhesus, mencionando que son equivalentes a las observadas en humanos con base a su morfología, solo que las latencias muestran valores más pequeños. Finalmente Möller y Burgess (1986), Laughlin y col (1999) e Ibáñez-Contreras y col (2011) mencionan la aparición de cuatro ondas definidas y constantes, observando que en la especie M. mulatta la onda más prominente y de mayor amplitud es la onda IV, siendo esta onda la más estable y la última en desaparecer al disminuir la intensidad del estímulo, por lo cual concluyen que la onda IV sería equivalente a la onda $\mathrm{V}$ en humanos que corresponde al colículo inferior, por el comportamiento de esta onda frente a los cambios de intensidad y morfología. Con base a lo anterior y de acuerdo a lo descrito por Alegre y col (2000) en este trabajo se evaluaron los intervalos I-II, II-IV y I-IV.

En el cuadro 3 se observa un alargamiento de los intervalos en los machos, principalmente en los intervalos II-IV y I-IV en relación a las hembras. Allen y Starr (1976), Velasco y col (1984) y Alegre y col (2000) reportan que el valor del intervalo I-IV en monos rhesus machos es de $2,90 \mathrm{mseg}$, concordando con lo reportado en este trabajo. Es importante resaltar que en la mayoría de los PEATC realizados en PNH los sujetos de estudio han sido machos, por lo que los resultados de las hembras expuestos en este trabajo ofrecen una nueva área de investigación y de estandarización de los PEATC en PNH de la especie M. mulatta.

Finalmente se observó el efecto de las diferentes intensidades empleadas $(70,50$ y $30 \mathrm{~dB})$ sobre el valor de las latencias absolutas, donde se observa que tanto en machos como en hembras se presenta la función latencia-intensidad (cuadro 2), la cual estipula que a mayor intensidad la latencia es más pequeña. Cabe destacar que los intervalos interondas no sufrieron variaciones en relación a las diferentes intensidades empleadas, tal como lo mencionan Elías (1997) y Soares y col (2010), indicando que solo las latencias interonda son estables frente a cambios en la intensidad del estímulo, por lo que pueden ser considerados como un indicador confiable en la evaluación audiológica y neurológica de los PEATC.

Este trabajo contribuye a la larga serie de estudios realizados a través de los PEATC en diversas especies animales y en humanos, donde se ha descrito que el registro de los PEATC es sensible a las diferencias en los parámetros de estimulación, a la escala de intensidad empleada y a las condiciones del registro. Los datos presentados en este estudio señalan la edad y el sexo como variables determinantes para establecer una normatividad en los parámetros de registro en esta especie de $\mathrm{PNH}$.

\section{RESUMEN}

El registro de los Potenciales Evocados Auditivos de Tallo Cerebral (PEATC) es sensible a las diferencias en los parámetros de estimulación, condiciones del registro y características de la especie utilizada, por lo que es imprescindible poseer valores de normalidad para cada laboratorio. El objetivo de este estudio fue obtener datos normativos acerca de los valores de latencia y de las respuestas electrofisiológicas de los diferentes componentes de los PEATC en el mono rhesus, en diferentes intensidades (70-30 dB). Se utilizaron 14 monos rhesus (Macaca mulatta) con una edad promedio de siete años, divididos en siete machos con un peso promedio de $7 \mathrm{~kg}$ y siete hembras con un peso aproximado de $5 \mathrm{~kg}$. Los PEATC se obtuvieron mediante la estimulación de los oídos con "clicks" de rarefacción a 70, 50 y $30 \mathrm{~dB}$ de intensidad. La actividad eléctrica cerebral fue recogida por medio de electrodos de disco, colocados en las derivaciones $\mathrm{Cz}(+)$, A1, A2 (-) y Fz como tierra, según el sistema 10/20 
internacional. Se observaron cuatro ondas constantes y definidas en las diferentes intensidades utilizadas, tanto en machos como en hembras. Debido a que no se encontraron diferencias significativas en t de Student por aferencias separadas, se unificaron las aferencias, evaluando las latencias absolutas de las cuatro ondas obtenidas, así como los intervalos I-II, II-IV y I-IV en los 14 sujetos, en las diferentes intensidades.

\section{AGRADECIMIENTOS}

Se agradece la participación del Psicólogo Sergio Reyes Pantoja, en la obtención y procesamiento de los datos para la realización de este trabajo.

\section{REFERENCIAS}

Alegre M, IG Gurtubay, J Iriarte, E Ciordia, M Manrique, J Artieda. 2001. Brainstem auditory evoked potentials (BAEPs) in the cynomolgus macaque monkey. Equivalence with human BAEPs and proposal of a new nomenclature. Hear Res 151, 115-120.

Allen AR, A Starr. 1978. Auditory brain stem potentials in monkey (M. mulatta) and man. Electroencephalogr Clin Neurophysiol 45, 53-63.

American Encephalographic Society. 1994. Guidelines on evoked potentials. J Clin Neurophysiology 11, 40-73.

Ankel-Simons F. 2000. Primate anatomy: An introduction. $2^{\text {nd }}$ ed. Academic Press, New York, USA.

Arai S. 2008. Brainstem auditory evoked potentials in cattle sedated with xylazine. Can J Vet Res 72, 287-290.

Bast TH, K Christensen, H Cummins, F Geist, C Hartman, M Hines. 1961. The anatomy of the Rhesus Monkey: Macaca mulatta. Hafner Publishing Co, New York, USA.

Delgado HJ, CF Zenker, JJ Barajas de Prat. 2003. Normalización de los potenciales evocados auditivos del tronco cerebral 1: Resultados en una muestra de adultos normoyentes. Revista Electrónica de Audiología 2, 13- 17.

DiPietro JA, KT Kiylighan, KA Costigan, SE Rubin, DE Shiffler, JL Henderson, JP Pillion. 2010. Prenatal antecedents of newborn neurological maturation. Child Dev 81, 115-130.

Doyle WJ, MM Saad, TJ Fria. 1983. Maturation of the auditory brain stem response in rhesus monkeys (Macaca mulatta). Electroencephalogr Clin Neurophysiol 56, 210-223.

Doyle WJ, DB Webster. 1991. Neonatal conductive hearing loss does not comprimese brainstem auditory function and structure in rhesus monkeys. Hear Res 54, 145-151.

Elías Y. 1996. Potenciales provocados auditivos del tallo cerebral en el diagnóstico del daño auditivo y neurológico del adulto. En: Hernández OA, Flores RT, Peñaloza LY (eds). Registros electrofisiológicos para el diagnóstico de la patología de la comunicación humana. Instituto de la Comunicación Humana. SSA, México, Pp 157-170.

Fowler CG, KB Chiasson, TH Leslie, D Thomas, TM Beasley, JW Kemnitz, R Weindruch. 2010. Auditory function in rhesus monkeys: effects of aging and caloric restriction in the Wisconsin monkeys five years later. Hear Res 261, 75-81.

Galván-Montaño A, B Hernández-Godínez, A Ibáñez-Contreras, E Cárdenas-Lesión, R Ramírez-Hernández, J Aragón-Inclán. 2010. Anesthetic management in intrauterine surgery to evaluate an experimental model of myelomeningocele in non human primates (Macaca mulatta). Acta Cir Bras 25, 294-297.

Gil-Carcedo LM. 2004. Otología. $2^{\mathrm{a}}$ ed. Editorial Médica Panamericana, Madrid, España.
Henry KR. 2004. Males lose hearing earlier in mouse models of lateonset age-related hearing loss; females lose hearing earlier in mouse models of early-onset hearing loss. Hear Res 190, 141-148.

Hultcrantz M, R Simonoska, AE Stenberg. 2006. Estrogen and hearing: a summary of recent investigations. Acta Otolaryngol 126, 10-14.

Ibáñez-Contreras, A Durand-Rivera, B Hernández-Godínez, SA ReyesPantoja, JJ Morales-Martínez. 2011. Potenciales evocados auditivos del tallo cerebral en monos rhesus (Macaca mulatta) en diferentes etapas fisiológicas en condiciones de cautiverio. Arch Med Vet 43, 223-232.

Kamada T, K Kameda, S Kojima. 1991. Auditory evoked potentials in the Japanese monkey. J Med Primatol 6, 284-289.

Lasky RE, MM Maier, EB Snodgrass, NK Laughlin, KE Hecox. 1995. Auditory evoked brainstem and middle latency responses in Macaca mulatta and humans. Hear Res 89, 212-225.

Laughlin NK, BK Hartup, RE Lasky, MM Meier, KE Hecox. 1999. The development of auditory even related potentials in the Rhesus Monkey (Macaca mulatta). Dev Psychobiol 34, 37-56.

López-Escámez JA, G Salguero, J Salinero. 1999. Age and sex differences in latencies of waves I, III and V in auditory brainstem response of normal hearing subjects. Acta Otorhinolaryngol Belg 53, 109-115.

McFadden SL, LW Henselman, XY Zheng. 1999. Sex differences in auditory sensitivity of chinchillas before and after exposure to impulse noise. Ear Hear 20, 164-174.

Møller AR, J Burgess. 1986. Neural generators of the brain-stem auditory evoked potentials (BAEPs) in the rhesus monkey. Electroencephalogr Clin Neurophysiol 65, 361-372.

Norma Oficial Mexicana. 1999. Especificaciones técnicas para la producción, cuidado y uso de los animales de laboratorio, NOM-062-ZOO-1999.

Pineda JA, TC Holmes, D Swick, SL Foote. 1989. Brain-stem auditory evoked potentials in squirrel monkey (Saimiri sciureus). Electroencephalogr Clin Neurophysiol 73, 532-543.

Populin LC. 2006. Monkey sound localization: head-restrained versus head-unrestrained orienting. J Neurosci 26, 9820-9832.

Recanzone GH. 2000. Response profiles of auditory cortical neurons to tones and noise in behaving macaque monkeys. Hear Res 150, 104-118.

Rhode WS, GL Roth, A Recio. 2010. Response properties of cochlear nucleus neurons in monkeys. Hear Res 259, 1-2.

Schneiderman E. 1992. Facial growth in the rhesus monkey. A longitudinal cephalometric study. Princeton University Press. New Jersey, USA.

SEMARNAT, Secretaría de Medio Ambiente y Recursos Naturales. 2007. Ley general de vida silvestre y su reglamento. Impresora y Encuadernado Progreso S.A., México DF, México.

Soares IA, PL Menezes, ATL Carnauba, LD Pereira. 2010. Standardization of brainstem auditory evoked potentials using a new device. Pro Fono 22, 421-426.

Torre III P, CG Fowler. 2000. Age- related changes in auditory function of rhesus monkeys (Macaca mulatta). Hear Res 142, 131-140.

Torre III P, RE Lasky, CG Fowler. 2000. Aging and middle ear function in Rhesus monkeys (Macaca mulatta). Audiology 39, 300-304.

Torre III P, JA Mattison, CG Fowler, MA Lane, GS Roth, DK Ingram. 2004. Assessment of auditory function in rhesus monkeys (Macaca mulatta): effects of age and calorie restriction. Neurobiol Aging 25, 945- 954.

Velasco M, F Velasco, R Romo, X Almanza. 1984. Subcortical correlates of the auditory brain stem potentials in the monkey: bipolar EEG and multiple unit activity responses. Int J Neurosci 22, 235-251.

Yadav A, OP Tandon, N Vaney. 2002. Auditory evoked responses during different phases of menstrual cycle. Indian J Physiol Pharmacol $46,449-456$. 